\title{
CORRELATION BETWEEN DEPRESSION AND ANXIETY AND THE LEVEL OF VITAMIN B12 IN PATIENTS WITH DEPRESSION AND ANXIETY AND HEALTHY CONTROLS
}

\author{
Aleksandar A. Todorov, \\ Petranka G. Chumpalova- \\ Tumbeva, \\ Maya Y. Stoimenova-Popova, \\ Vanya S. Popova ${ }^{1}$, \\ Doroteya K. Todorieva- \\ Todorova ${ }^{1}$, \\ Nikolai T. Tzvetkov ${ }^{1}$, \\ Ivailo G. Hristov ${ }^{1}$, \\ Georgi K. Georgiev, \\ Valentin I. Valtchev ${ }^{2}$, \\ Niya A. Krasteva ${ }^{3}$, \\ Ralitza G. Ilieva ${ }^{4}$, \\ Emiliya M. Dimitrova, \\ Ljudmil Z. Tumbev, \\ Adelaida L. Ruseva ${ }^{5}$
}

Department of Psychiatry and Medical Psychology,

Medical University - Pleven

${ }^{1}$ Department of Nephrology,

Haematology and Gastroenterology, Medical University - Pleven

${ }^{2}$ Department of Physiology, National Sports Academy - Sofia ${ }^{3}$ Department of Children's Diseases, Medical University - Pleven ${ }^{4}$ Hematology Clinic, University Hospital - Pleven

${ }^{5}$ Department of Immunology, Allergology and Clinical Laboratory, Medical University - Pleven,

\section{Corresponding Author:}

Aleksandar A.Todorov

Department of Psychiatry and Medical

Psychology,

Medical University - Pleven

113, Storgozia,

Pleven, 5800

Bulgaria

e-mail:alex_9020@abv.bg

Received: December 22, 2017

Revision received: February 6, 2018

Accepted: February 27, 2018

\section{Summary}

Affective disorders, including depression, are of great social importance and lead to serious everyday life infringement and disability. Affective disorders are one of the main causes of suicide causes. Anxiety disorders represent a variety of psychic disorders that often lead to disability. Anxiety and depression syndromes together are often seen in patients. Vitamin B12 (cobalamin) is the only vitamin containing cobalt. Our aim was to investigate, evaluate and compare depression and increased anxiety and serum Vitamin B12 level in patients with depression, in patients with Vitamin B12 deficiency anemia and healthy controls. We investigated 74 subjects -38 patients and 36 healthy controls. Serum Vitamin B12 level was measured in all participants. It is assumed that normal ranges of Vitamin B12 level vary. The most recently accepted ones are 200 to $900 \mathrm{pg} / \mathrm{ml}$. In cases of levels below $200 \mathrm{pg} / \mathrm{ml}$, a therapy with vitamin B12 should be applied. On the other hand, the level necessary for normal biochemical processes is higher $-250 \mathrm{pg} / \mathrm{ml}$. In our study, serum Vitamin B12 level in more than $50 \%$ of patients with depression/anxiety was below $200 \mathrm{pg} / \mathrm{ml}$, and in more than $60 \%$ of these patients it was below $250 \mathrm{pg} / \mathrm{ml}$.

Key words: depression, anxiety, B12 deficiency anemia

\section{Introduction}

The terms "depression" and "anxiety" are often used nowadays. Their range of meaning varies a lot - starting from mild and trivial, to serious and life threatening.

Affective disorders, depression included, are diseases of great social importance that lead to serious everyday life infringement and disability. The lifetime prevalence of depression is $5-7 \%$ in nosological epidemiology with no age limits $[1,2]$. The onset is in younger age, and in $33 \%$ of the patients it is chronic [3]. Important disease features are high suicidal risk and social functioning disturbance. Somatic diseases are often accompanied by depression symptoms. As far as sickness and disability are concerned, depression represents about 11\% [3]. According to the World Health Organization (WHO) data, in only a few years depression will take second place as a cause for disability and a leading place in countries with high life standards, and will account for a great economic and social capital loss in every society [1-6]. 
Affective disorders are among the main suicidal causes [7]. In highly developed countries, when an affective disorder is diagnosed, suicide is the second cause for death after ischemic heart disease [2]. The suicide rate among patients with psychotic disorders is about $77-92 \%$. Thirty percent of them are due to affective disorders. Depression is defined as the main suicide cause [7].

Anxiety is a psychological term to define an emotional state, characterized by an insecure feeling and worry. Anxiety is known for the lack of clear current threat. Fear is well distinguished from anxiety, as fear is targeted. Anxiety appears and increases, regardless of local environment changes. It is one of the mechanisms for the organism to point out the necessity for reaction preparation, when a situation changes and extreme physical and psychological tension is present, the so-called normal anxiety [5]. However, in pathological anxiety there are some features that define it as a disorder symptom. It is connected to past or present experience and is formed by one's view and unconscious internal conflicts; stereotyped and repeated [3, 5]. Anxiety disorders represent a group of various conditions that often lead to disability. Anxiety disorders are diagnosed in about one-fifth of all outpatient psychiatric examinations/consultations [3]. It is a bad tendency that only $1.7 \%$ of affected people seek and get psychiatric help, which leads to chronic and worsened conditions [3]. Moreover, when properly diagnosed and treated, a patient could function again as before [3]. The autonomy reaction dysregulation increases the risk for somatic diseases onset - cardiovascular, gastrointestinal, etc. There are 4 pathological anxiety variants: as a response to a life event, as a part of mental disorder, as a separate anxiety syndrome, or as a part of a somatic disease symptoms complex [3]. In the International Classification of Diseases 10th revision (ICD10) we find a variety of disorders with a leading anxiety syndrome.

A combination of anxiety and depression syndromes is often seen in patients. Etiologically, there are 3 models for their simultaneous appearance: a primary anxiety disorder, not treated or not diagnosed, that leads to secondary depression; secondary anxiety as a result of primary chronic depression; both appearing as a reaction to a common stressor.
Vitamin B12 plays an important role in the pathogenesis of depression and anxiety. It is not produced by the human organism and is taken with food. Vitamin B12 (cobalamin) is the only vitamin that contains cobalt. Vitamin B12 is produced in the gastrointestinal tract of animals and the only one that cannot be found in plants. Plants do not produce it but some investigators mention that pseudo-Vitamin B12 is found in the spirulina and tempeh, and the pseudo B12 blocks the real vitamin and deactivates it. Foods that contain vitamin B12 are chicken meat, fish, milk products, and eggs [8].

The most common clinical manifestation of vitamin B12 deficiency is megaloblastic anemia (anemia perniciosa), caused by decreased vitamin B12 absorption. In its pathogenesis, one of the two coenzymes forms of vitamin B12 (methylcobalamin), plays a leading role in two key biochemical reactions. Methylcobalamin is a co-factor of the methyltransferase enzyme in homocysteine conversion to methionine. The biochemical reactions then continue with deoxyribonucleic acid (DNA) synthesis. It is damaged when vitamin B12 is deficient - the nucleus is not ready for cell division (DNA duplication). Methionine is necessary for S-adenosylmethionine (SAM) synthesis as it is used for myelin synthesis. SAM is a donor of methyl group in many biochemical reactions, including the brain (the cause for neurological and psychic symptoms in vitamin B12 deficiency). Another co-enzyme form is 5-deoxyadenosilcobalamin that is a co-factor for methylmalonyl coenzyme A (CoA) mutase in the conversion of L-methylmalonyl CoA to succinyl CoA. This reaction is an important stage in many oxidative processes. No folic acid is required. The deficiency of the second co-enzyme form also blocks the conversion and is responsible for neurological symptoms (increased level of methylmalonic and propionic acid) [8].

As a part of scientific projects No 22/2016, funded by Medical University - Pleven, a study was conducted on the association between Vitamin B12 level and depression/anxiety.

Our aim was to investigate, evaluate and compare depression and increased anxiety and serum vitamin B12 level in patients with depression, in patients with vitamin B12 deficiency anemia, and healthy controls.

The scientific hypothesis was that there 
is a correlation between depression/anxiety symptoms and serum vitamin B12 level.

Objectives:

- To measure vitamin B12 level in the blood of the patients with depression and anxiety;

- To evaluate the level of depression and anxiety in the patients with clinically and laboratory proven vitamin B12 deficiency anemia;

- To form a healthy control group with no clinical data about anxiety and depression. To evaluate their level of depression/anxiety and to measure their vitamin B12 level;

- To analyze a possible correlation between depression and anxiety and vitamin B12 deficiency as two separate manifestations of decreased vitamin B12 levels in the blood according to the results.

\section{Materials and Methods}

Period: November 2016 - June, 2017.

We investigated 74 persons - 38 patients (from Psychiatry clinic) and 36 healthy controls. The patients' group included out-patient seen in First and Second Psychiatry Clinic. Healthy controls were randomly selected subjects with no medical history of anemia, affective or anxiety disorder. All participants signed an informed consent form.

Our activities were:

- To form two groups: patients with depression and/or anxiety and healthy controls;

- To assess depression/anxiety, by psychic interview and standard questionnaires - Hamilton anxiety score (HAM-A), Hamilton depression score (HAM-D), Montgomery-Asberg Depression Rating Scale (MADRS). Psychometric investigations were done in the first 5 days after admission;

- To measure serum vitamin B12 level in all participants;

- To group criteria: Criteria for inclusion and exclusion were given in Table 1.

Table 1. Criteria for inclusion/exclusion of patients and healthy controls

\begin{tabular}{lll}
\hline Group & Inclusion criteria & Exclusion criteria \\
\hline Depression/anxiety & older than 18, equal for both genders & younger than 18 \\
\hline & history for depression/anxiety & other psychiatric disorders \\
\hline Vitamin B12 deficiency anemia & equal for both genders & younger than 18 \\
\hline & clinical and laboratory data for vitamin & $\begin{array}{l}\text { other hematological disease or } \\
\text { anemia of an unknown cause }\end{array}$ \\
\hline Healthy control group & older than 18, equal for both genders & younger than 18 \\
\hline & & $\begin{array}{l}\text { depression/anxiety disorder, } \\
\text { macrocytic anemia confirmed }\end{array}$ \\
\hline
\end{tabular}

The age range of $70 \%$ of the patients was 18 to 64 years. We collected 5-7 $\mathrm{ml}$ blood from each patient. A questionnaire was filled containing information about disease onset, medical history and past depression/anxiety episodes.

Data were entered into an Excel table and was processed statistically with STATGRAPHICS. We used linear regression analysis. A value $\mathrm{p}<0.05$ was considered as a threshold of statistical significance.

\section{Results}

The patients with depression/anxiety were 38 - 15 males and 23 females (age range 29 to 77 years, mean age 54.16, standard deviation 13.43). The mean level of Vitamin B12 in all patients was $259.22 \mathrm{pg} / \mathrm{ml}(248.39 \mathrm{pg} / \mathrm{ml}$ in males and $266.28 \mathrm{pg} / \mathrm{ml}$ in females). Psychometric and clinical data of all patients showed increased levels of depression and anxiety. 
- Todorov A, et al. Correlation between depression and anxiety and the level of vitamin b12 ...

The healthy control group included 36 subjects - 14 males and 22 females, aged 19 to 79 , mean age 41.83 years (Table 2). Their mean Vitamin B12 serum level was $365.68 \mathrm{pg} /$ $\mathrm{ml}(369.65 \mathrm{pg} / \mathrm{ml}$ in males and $363.15 \mathrm{pg} / \mathrm{ml}$ in females). The psychometric evaluation did not show any significant increase in anxiety and depression level.

Table 2. Mean age and level of vitamin $B_{12}$ of patients and healthy controls

\begin{tabular}{llcc}
\hline Group & $\mathrm{N}$ & Mean age & Mean Vitamin B12 level \\
\hline Patients & $\mathbf{3 8}$ & $\mathbf{5 4 . 2}$ & $\mathbf{2 5 9 . 2 2}$ \\
\hline Males & 15 & & 248.39 \\
\hline Females & 23 & & 266.28 \\
\hline Healthy controls & $\mathbf{3 6}$ & $\mathbf{4 1 . 8}$ & $\mathbf{3 6 5 . 6 8}$ \\
\hline Males & 14 & & 369.65 \\
\hline Females & 22 & & 363.15 \\
\hline
\end{tabular}

When statistical software was used, a correlation between Vitamin B12 level and psychometric tests became obvious. By using a linear model of regression analysis we proved a correlation between Vitamin B12 concentration and psychometric evaluation. The correlational coefficient was -0.32 (correlation between Vitamin B12 level and HAM-A score) and -0.47 (correlation between Vitamin B12 level and MADRS score), and this was shown on Figure 1 and Figure 2.

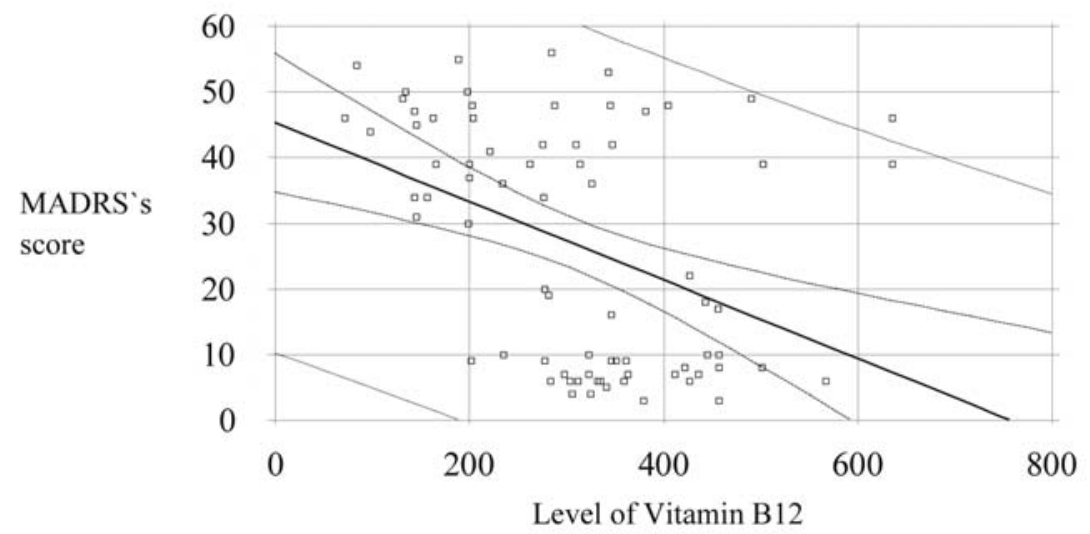

Figure 1. Model of correlation between level of vitamin B12 and HAM-A score (HAM-A=21.6-0.017*B12; $\mathrm{p}=0.005$ )

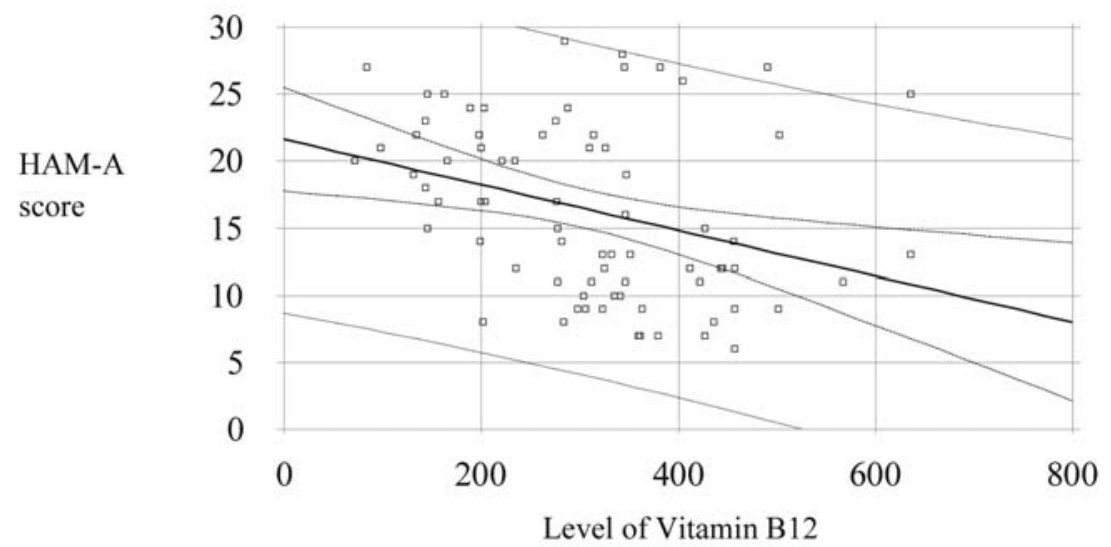

Figure 2. Model of correlation between level of vitamin B12 and MADRS`s score (MADRS=45.3-0,06*B12; $\mathrm{p}=0.001)$ 


\section{Discussion}

Psychiatric symptoms, mainly depression and anxiety, and their association to Vitamin B12 level have been known since 1950. During the years, much new information has been found about the biochemical transformation, which that explains this correlation. According to literature data, 10 to $30 \%$ of patients with depression have low Vitamin B12 levels [9-11]. In the recent decade, studies worldwide have reported a more effective therapy for patients with depression with Vitamin B12 (1 mg daily), and also with a combination of an antidepressant and Vitamin B12 [12, 13].

There is data in the literature that $27 \%$ of females diagnosed with depression have low levels of Vitamin B12 [13]. Adding it to their therapy helps to reduce depression symptoms. L. Barclay (2010) reported a study including 3503 patients, older than 65 . He evaluated their depression symptoms for 12 years. The results showed that frequency and depression degree decreased by $2 \%$ annually when the level of Vitamin B12 was $10 \mu \mathrm{g}$ higher, thus proving it plays a role in depression and anxiety prophylaxis [14]. A Finnish study, conducted in 2003, showed that 115 patients who had been treated for depression would benefit from higher levels of Vitamin B12. Different studies point out that $30 \%$ of patients hospitalized for depression have cobalamin deficiency [12].

Many authors describe serum level changes as a certain proof for Vitamin B12 influence on hematopoiesis and psychic processes. Normal ranges of Vitamin B12 level vary but the most recently accepted are 200 to $900 \mathrm{pg} / \mathrm{ml}$. If below 200pg/ml, a therapeutic Vitamin B12 should be administered. On the other hand, the concentration necessary for normal biochemical processes is higher than $250 \mathrm{pg} / \mathrm{ml}$.

Our results prove our hypothesis and support data from other studies, confirming that Vitamin B12 level determines the degree of depression/ anxiety: the mean Vitamin B12 level in the patients' group was lower than in healthy controls.

Some correlations are still not clear:

- A quality correlation between serum Vitamin B12 level and degree of depression/anxiety;
- The therapeutic effect of Vitamin B12 in cases of depression/anxiety.

\section{Conclusions}

The serum level of Vitamin B12 plays an important role in depression/anxiety development and clinical manifestation.

The scientific hypothesis for lower levels in our patients with depression/anxiety, as compared to healthy controls, was confirmed. The data obtained proved that Vitamin B12 level in more than $50 \%$ of patients with depression/ anxiety was below $200 \mathrm{pg} / \mathrm{ml}$, and in more than $60 \%$ of these patients it was below $250 \mathrm{pg} / \mathrm{ml}$; i.e. the levels needed for normal functioning.

The correlation between depression and Vitamin B12 deficiency is an actual problem because it is a common condition with serious medical, social and financial consequences and a poor prognosis. Vitamin B12 was found to be an effective option for treatment and prophylaxis of depression/anxiety.

\section{Acknowledgements}

This study was supported by Medical University - Pleven (Project No22/2016)

\section{References}

1. Kaplan HI, Sadock BJ. Kaplan. and Sadock's synopsis of psychiatry: behavioral sciences, clinical psychiatry. 11th ed. London:Wolters Kluwer;2015.

2. Andersan NC, Black DW. Introductory textbook of psychiatry. 3rd ed. London: American psychiatric publishing. Inc; 2001.

3. Milanova VK. [Psychiatry - book for medical student and medical specialists].1st ed. Sofia: Medicine and P.T;2013. Bulgarian.

4. Akabaliev V. [Book for medical student]. 3rd ed. Plovdiv: Laks book;2015. Bulgarian.

5. Boncheva I. [Medical psychology - book for health care specialists]. Varna: Medical University;2012. Bulgarian.

6. Madzhirova N. [Practical Guide of psychiatry for general medicine specialists]. Sofia: Rajkov; 2009. Bulgarian.

7. Stoimenova M. [The suicide - an escape or a decision?] Sofia: Dita-M; 2014. Bulgarian.

8. Todorov V, Ljutckanova E, Marinova I, Lyubomirova D, Tzvetkov N. [Internal diseases 
- Todorov A, et al. Correlation between depression and anxiety and the level of vitamin b12 ...

2nd part - book for medical students]. 1st ed. Pleven: Medical University; 2012. Bulgarian.

9. Morris $\mathrm{AL}$, Thomas $\mathrm{AB}$, Francis JK, Jerome L, Loren RM, Richard W. Megavitamin and orthomolecular therapy in psychiatry. Washington: American Psychiatric Association; 1973.

10. Airola P. Hypoglycemia: A Better Approach. Phoenix, Ariz.: Health Plus Publishers; 1977.

11. Miller C. Vitamin B12 for depression. Livestrong.com [Internet].c2017 [cited 2017 Sept] [about 1p.] Available from: https://www. livestrong.com/article/463325-vitamin-b12-fordepression/
12. Coppen A, Bolander-Gouaille C. Treatment of depression: time to consider folic acid and vitamin B12. J Psychopharmacol. 2005;19(1):59-65.

13. Methylcobalamininfo Info. Treating depression with vitamin B12. [Internet]. c2015 [cited 2017, Sept22]; [about 1 screen.] Available from: http://www.methylcobalamininfo.com/b12depression-treatment/

14. Barclay L. Vitamins B6, B12 may protect against depression in older adults. Medscape [Internet] 2010; [cited 2017 Sept 12]; [about 1 p.] Available from: https://www.medscape.com/ viewarticle/724456. 